\title{
ON THE JOINT SPECTRA OF DOUBLY COMMUTING $n$-TUPLES OF SEMI-NORMAL OPERATORS
}

\author{
by MUNEO CHŌ and A. T. DASH
}

(Received 21 September, 1983)

Let $H$ be a complex Hilbert space. For any operator (bounded linear transformation) $T$ on $H$, we denote the spectrum of $T$ by $\sigma(T)$. Let $T=\left(T_{1}, \ldots, T_{n}\right)$ be an $n$-tuple of commuting operators on $H$. Let $\operatorname{Sp}(T)$ be the Taylor joint spectrum of $T$. We refer the reader to [8] for the definition of $\operatorname{Sp}(T)$. A point $\nu=\left(\nu_{1}, \ldots, \nu_{n}\right)$ of $\mathbb{C}^{n}$ is in the joint approximate point spectrum $\sigma_{\pi}(T)$ of $T$ if there exists a sequence $\left\{x_{k}\right\}$ of unit vectors in $H$ such that

$$
\left\|\left(T_{j}-\nu_{j}\right) x_{k}\right\| \rightarrow 0 \text { as } k \rightarrow \infty \text { for } j=1, \ldots, n .
$$

A point $\nu=\left(\nu_{1}, \ldots, \nu_{n}\right)$ of $\mathbb{C}^{n}$ is in the joint approximate compression spectrum $\sigma_{\delta}(T)$ of $T$ if there exists a sequence $\left\{x_{k}\right\}$ of unit vectors in $\boldsymbol{H}$ such that

$$
\left\|\left(T_{j}-\nu_{j}\right)^{*} x_{k}\right\| \rightarrow 0 \text { as } k \rightarrow \infty \text { for } j=1, \ldots, n \text {. }
$$

A point $\nu=\left(\nu_{1}, \ldots, \nu_{n}\right)$ of $\mathbb{C}^{n}$ is in the joint point spectrum $\sigma_{p}(T)$ of $T$ if there exists a non-zero vector $x$ in $H$ such that $\left(T_{j}-\nu_{j}\right) x=0$ for all $j, 1 \leqslant j \leqslant n$.

Consult [4] for further details regarding the notions of $\sigma_{\pi}(T), \sigma_{\delta}(T)$ and $\sigma_{p}(T)$. It is well known that $\sigma_{\pi}(T) \cup \sigma_{\delta}(T) \subseteq \mathrm{Sp}(T)$.

LeMmA 1 (S. K. Berberian [1]). Let $\mathrm{B}(H)$ be the *-algebra of all bounded operators on $H$. Then there exists an extension space $K$ of $H$ and a faithful ${ }^{*}$-homomorphism of $B(H)$ into $B(K): S \rightarrow S^{0}$ such that

$$
\sigma_{\pi}(S)=\sigma_{\pi}\left(S^{0}\right)=\sigma_{p}\left(S^{0}\right) .
$$

Furthermore, if $T=\left(T_{1}, \ldots, T_{n}\right)$ is an $n$-tuple of commuting operators on $H$ then

$$
\sigma_{\pi}\left(T_{1}, \ldots, T_{n}\right)=\sigma_{\pi}\left(T_{1}^{0}, \ldots, T_{n}^{0}\right)=\sigma_{p}\left(T_{1}^{0}, \ldots, T_{n}^{0}\right) .
$$

See [2] or [5, Proposition 3.2] for a proof.

An $n$-tuple $T=\left(T_{1}, \ldots, T_{n}\right)$ of operators is said to be hyponormal if $T_{j}^{*} T_{j}-T_{j} T_{i}^{*} \geqslant 0$ for $j=1, \ldots, n$. We say that $T=\left(T_{1}, \ldots, T_{n}\right)$ is semi-normal if each $T_{i}$ is semi-normal $\left(T_{i}\right.$ or $T_{i}^{*}$ is hyponormal) for $j, 1 \leqslant j \leqslant n$.

LEMMA $2\left[3\right.$, Corollary 3.8]. Let $T=\left(T_{1}, \ldots, T_{n}\right)$ be a doubly commuting $n$-tuple of hyponormal operators (i.e. $T_{i} T_{i}=T_{i} T_{i}$ for all $i, j$ and $T_{i} T_{i}^{*}=T_{i}^{*} T_{i}$ for all $i \neq j$, and $\left.T_{i}^{*} T_{i} \geqslant T_{i} T_{i}^{*}\right)$. Then

$$
\operatorname{Sp}(T)=\sigma_{\delta}(T) .
$$

Furthermore, if $T=\left(T_{1}, T_{2}\right)$ is a doubly commuting pair of operators with $T_{1}$ and $T_{2}^{*}$ being hyponormal then

$$
\left(\nu_{1}, \nu_{2}\right) \in \operatorname{Sp}\left(T_{1}, T_{2}\right) \text { if and only if }\left(\nu_{1}, \nu_{2}^{*}\right) \in \operatorname{Sp}\left(T_{1}, T_{2}^{*}\right)=\sigma_{\delta}\left(T_{1}, T_{2}^{*}\right) .
$$

Glasgow Math. J. 26 (1985) 47-50. 
The following result was proved by Putman [6]. See also [7].

LEMMA 3. Let $T$ be a semi-normal operator with $T=A+i B$.

(i) If $\lambda \in \sigma(A)$, there exists a real number $\mu$ and a sequence $\left\{x_{k}\right\}$ of unit vectors such that

$$
\left\|(A-\lambda) x_{k}\right\| \rightarrow 0 \text { and }\left\|(B-\mu) x_{k}\right\| \rightarrow 0,
$$

that is, $\lambda+i \mu \in \sigma(T)$. Similarly, if $\mu \in \sigma(B)$ then there exists a real number $\lambda$ and $\left\{x_{k}\right\}$ in $H$ with $\left\|x_{k}\right\|=1$ such that

$$
\left.\left\|(A-\lambda) x_{k}\right\| \rightarrow 0 \text { and } \|(B-\mu) x_{k}\right) \| \rightarrow 0 .
$$

(ii) Let $\lambda$ and $\mu$ be real numbers. If $\lambda+i \mu \in \sigma(T)$ then $\lambda \in \sigma(A)$ and $\mu \in \sigma(B)$.

We generalize this result to doubly commuting $n$-tuples of semi-normal operators.

THEOREM. Let $T=\left(T_{1}, \ldots, T_{n}\right)$ be a doubly commuting $n$-tuple of semi-normal operators with $T_{j}=A_{j}+i B_{j}, j=1, \ldots, n$. Write $A=\left(A_{1}, \ldots, A_{n}\right)$ and $B=\left(B_{1}, \ldots, B_{n}\right)$.

(i) If $\lambda=\left(\lambda_{1}, \ldots, \lambda_{n}\right) \in \operatorname{Sp}(A)$ then there exists $\mu=\left(\mu_{1}, \ldots, \mu_{n}\right) \in \mathbb{R}^{n}$ and a sequence $\left\{x_{k}\right\}$ of unit vectors in $H$ such that

$$
\left\|\left(A_{j}-\lambda_{j}\right) x_{k}\right\| \rightarrow 0 \quad \text { and } \quad\left\|\left(B_{j}-\mu_{j}\right) x_{k}\right\| \rightarrow 0, \quad j=1, \ldots, n,
$$

that is, $\nu=\lambda+i \mu=\left(\lambda_{1}+i \mu_{1}, \ldots, \lambda_{n}+i \mu_{n}\right) \in \operatorname{Sp}(T)$.

An analogous result holds for $\operatorname{Sp}(B)$.

(ii) Let $\lambda_{j}$ and $\mu_{j}$ be real numbers for $j, j=1, \ldots, n$. If $\lambda+i \mu=$ $\left(\lambda_{1}+i \mu_{1}, \ldots, \lambda_{n}+i \mu_{n}\right) \in \operatorname{Sp}(T)$ then $\lambda=\left(\lambda_{1}, \ldots, \lambda_{n}\right) \in \operatorname{Sp}(A)$ and $\mu=\left(\mu_{1}, \ldots, \mu_{n}\right) \in$ $\mathrm{Sp}(B)$.

Proof. It is clear from Lemma 2 (2) and the proof of the theorem given below that there is no loss of generality in assuming that $T=\left(T_{1}, \ldots, T_{n}\right)$ is hyponormal.

(i) Here we give the proof for $\operatorname{Sp}(A)$. The proof for $\operatorname{Sp}(B)$ is similar. Furthermore, since $A$ is an $n$-tuple of commuting self-adjoint operators, it is well known that $\operatorname{Sp}(A)=\sigma_{\pi}(A)$. (Consult [4] or Lemma 2.) Thus $\lambda \in \sigma_{\pi}(A)$. By Lemma 1, we have $\sigma_{\pi}(A)=\sigma_{\pi}\left(A^{0}\right)=\sigma_{p}\left(A^{0}\right)$. Hence $\lambda=\left(\lambda_{1}, \ldots, \lambda_{n}\right) \in \sigma_{p}\left(A^{0}\right)$. Set

$$
M=\left\{f \in K:\left(A_{i}^{0}-\lambda_{j}\right) f=0, \quad j=1, \ldots, n\right\} .
$$

We show that $M$ is a reducing subspace for $B_{1}^{0}, \ldots, B_{n}^{0}$. Since $T_{i}^{0}$ is hyponormal, we have $T_{j}^{0 *} T_{i}^{0}-T_{j}^{0} T_{i}^{0 *}=2 i\left(A_{j}^{0} B_{i}^{0}-B_{j}^{0} A_{j}^{0}\right) \geqslant 0$. Set $C_{j}=i\left(A_{j}^{0} B_{j}^{0}-B_{j}^{0} A_{j}^{0}\right)$. Thus $C_{j} \geqslant 0$. But $\left(A_{j}^{0}-\lambda_{j}\right) B_{j}^{0}-B_{j}^{0}\left(A_{j}^{0}-\lambda_{j}\right)=-i C_{j}$. Therefore, for $f \in M,-i\left(C_{i} f, f\right)=\left(\left(A_{j}^{0}-\lambda_{j}\right) B_{j}^{0} f, f\right)-$ $\left(B_{j}^{0}\left(A_{j}^{0}-\lambda_{j}\right) f, f\right)=0$. Since $C_{j} \geqslant 0$, it follows that $C_{j} f=0$. This implies that $\left(A_{j}^{0}-\lambda_{j}\right) B_{j}^{0} f=$ 0 . If $i \neq j,\left(A_{j}^{0}-\lambda_{j}\right) B_{i}^{0}=B_{i}^{0}\left(A_{j}^{0}-\lambda_{j}\right)$. Thus $\left(A_{j}^{0}-\lambda_{j}\right) B_{i}^{0} f=0$ for $f \in M$. Hence $M$ is a reducing subspace for $B_{1}^{0}, \ldots, B_{n}^{0}$. Thus there exists $\mu=\left(\mu_{1}, \ldots, \mu_{n}\right) \in \sigma_{\pi}\left(B_{\left.1\right|_{M}}^{0}, \ldots, B_{\left.n\right|_{M}}^{0}\right)=$ $\sigma_{p}\left(B_{\left.1\right|_{M}}^{0}, \ldots, B_{\left.n\right|_{M}}^{0}\right)$. This implies that there exists a non-zero vector $f \in M$ such that $B_{j}^{0} f=$ $\mu_{j} f, j=1, \ldots, n$. Hence $(\lambda, \mu) \in \sigma_{p}\left(A^{0}, B^{0}\right)=\sigma_{\pi}(A, B)$. Therefore, there exists $\left\{x_{k}\right\}$ in $H$ with $\left\|x_{k}\right\|=1$ such that

$$
\left\|\left(A_{j}-\lambda_{j}\right) x_{k}\right\| \rightarrow 0 \quad \text { and } \quad\left\|\left(B_{j}-\mu_{j}\right) x_{k}\right\| \rightarrow 0, \quad j=1, \ldots, n .
$$


(ii) We will prove this part of the theorem by the method of induction. For $n=1$, see Lemma 3(ii). However, we give here a simple proof. If $\nu=\lambda+i \mu \in \sigma(T)$, where $T$ is hyponormal, then there exists a real number $\mu^{\prime}$ such that $\nu^{\prime}=\lambda+i \mu^{\prime} \in \partial \sigma(T)$. Here $\partial \sigma(T)$ denotes the boundary of $\sigma(T)$. But $\partial \sigma(T) \subseteq \sigma_{\pi}(T)$. This means that there exists a sequence $\left\{x_{k}\right\}$ of unit vectors in $H$ such that $\left(\left(T-\nu^{\prime}\right)^{*}\left(T-\nu^{\prime}\right) x_{k}, x_{k}\right) \rightarrow 0$. But $\left(T-\nu^{\prime}\right)^{*}\left(T-\nu^{\prime}\right)=(A-\lambda)^{2}+(B-\mu)^{2}+C$. Since $C \geqslant 0$, this implies that $\left\|(A-\lambda) x_{k}\right\| \rightarrow 0$. Thus $\lambda \in \sigma(A)$. Next we assume that the theorem is true for $(n-1)$-tuples $\left(T_{1}, \ldots, T_{n-1}\right)$. Moreover, if we denote the complex conjugate of a complex number $\nu$ by $\nu^{*}$, then $\operatorname{Sp}(T)=\sigma_{\delta}(T)=\sigma_{\pi}\left(T^{*}\right)^{*}=\sigma_{\pi}\left(T^{* 0}\right)^{*}=\sigma_{p}\left(T^{* 0}\right)^{*}$. Thus $\nu^{*}=\left(\nu_{1}^{*}, \ldots, \nu_{n}^{*}\right) \in \sigma_{\mathrm{p}}\left(T^{* 0}\right)$. Hence there exists $f \in K$ such that $\left(T_{j}^{* 0}-\nu_{j}^{*}\right) f=0$ for $j=1, \ldots, n$. Let $E=$ $\left\{f \in K:\left(T_{n}^{* 0}-v_{i}^{*}\right) f=0\right\}$. Since $T_{1}^{0}, \ldots, T_{n-1}^{0}$ are doubly commuting, it is clear that $E$ is a reducing subspace of $T_{1}^{0}, \ldots, T_{n-1}^{0}$. Thus $\left(\nu_{1}^{*}, \ldots, \nu_{n-1}^{*}\right) \in \sigma_{p}\left(T_{\left.1\right|_{E}}^{* 0}, \ldots, T_{n-\left.1\right|_{F}}^{* 0}\right)$. This implies that $\left(\nu_{1}, \ldots, \nu_{n-1}\right) \in \operatorname{Sp}\left(T_{\left.1\right|_{E}}^{0}, \ldots, T_{n-\left.1\right|_{E}}^{0}\right)$. Note that $\left(T_{\left.1\right|_{E}}^{0}, \ldots, T_{n-\left.1\right|_{E}}^{0}\right)$ is a doubly commuting $(n-1)$-tuple of hyponormal operators. Thus by the assumption, it follows that $\left(\lambda_{1}, \ldots, \lambda_{n-1}\right) \in \sigma_{\pi}\left(A_{\left.1\right|_{E}}^{0}, \ldots, A_{n-\left.1\right|_{E}}^{0}\right)=\sigma_{p}\left(A_{1_{\mid E}}^{0}, \ldots, A_{n-\left.1\right|_{E}}^{0}\right)$. Therefore, there exists a nonzero vector $f_{0}$ such that

$$
A_{i}^{o} f_{0}=\lambda_{j} f_{0} \quad(j=1, \ldots, n-1) \quad \text { and } \quad T_{n}^{* 0} f_{0}=\nu_{n}^{*} f_{0}
$$

Let $N=\left\{f \in K: A_{j}^{0} f=\lambda_{j} f, j=1, \ldots, n-1\right\}$. Thus, by equation (3), we have $\nu_{n} \in \sigma\left(T_{\left.n\right|_{N}}^{0}\right)$. Set $\nu_{n}=\lambda_{n}+i \mu_{n}$. Clearly $T_{\left.n\right|_{N}}^{0}$ is hyponormal. But $\partial \sigma\left(T_{\left.n\right|_{N}}^{0}\right) \subseteq \sigma_{\pi}\left(T_{\left.n\right|_{N}}^{0}\right)$. Thus there exists a real number $\mu_{n}^{\prime}$ such that $\nu_{n}^{\prime}=\lambda_{n}+i \mu_{n}^{\prime} \in \partial \sigma\left(T_{\left.n\right|_{N}}^{0}\right) \subseteq \sigma_{\pi}\left(T_{\left.n\right|_{N}}^{0}\right)=\sigma_{p}\left(T_{\left.n\right|_{N}}^{0}\right)$. This means that there exists $f \in N$ such that $\left(T_{n}^{0}-\nu_{n}^{\prime}\right) f=0$. But $T_{\left.n\right|_{N}}^{0}$ is hyponormal and hence $T_{n \|_{N}}^{0}-\nu_{n}^{\prime}$ is hyponormal. Thus $\left.0=\left(T_{\left.n\right|_{N}}^{0}-\nu_{n}^{\prime}\right)^{*}\left(T_{\left.n\right|_{N}}^{0}-\nu_{n}^{\prime}\right) f=\left(A_{\left.n\right|_{N}}^{0}-\lambda_{n}\right)^{2} f+B_{\left.n\right|_{N}}^{0}-\mu_{n}^{\prime}\right)^{2} f+C_{n} f$. Since $C_{n} \geqslant 0$, this implies that $\left(A_{n \mid N}^{0}-\lambda_{n}\right) f=0$. Therefore, we have $\left(A_{j}^{0}-\lambda_{j}\right) f=0$, for $j=1, \ldots, n$. Hence $\lambda \in \sigma_{p}\left(A_{1}^{0}, \ldots, A_{n}^{0}\right)=\sigma_{\pi}\left(A_{1}, \ldots, A_{n}\right)$. Similarly, one shows that $\left(\mu_{1}, \ldots, \mu_{n}\right) \in$ $\sigma_{\pi}\left(B_{1}, \ldots, B_{n}\right)$. This proves the theorem.

Thus we have shown:

and

$$
\operatorname{Sp}(A)=\{\operatorname{Re} \nu: \nu \in \operatorname{Sp}(T)\}
$$

$$
\operatorname{Sp}(B)=\{\operatorname{Im} \nu: \nu \in \operatorname{Sp}(T)\}
$$

where $T=\left(T_{1}, \ldots, T_{n}\right)$ is an $n$-tuple of doubly commuting semi-normal operators, and $\operatorname{Re} \nu=\left(\operatorname{Re} \nu_{1}, \ldots, \operatorname{Re} \nu_{n}\right)$ and $\operatorname{Im} \nu=\left(\operatorname{Im} \nu_{1}, \ldots, \operatorname{Im} \nu_{n}\right)$.

CoRollary. Let $T=\left(T_{1}, \ldots, T_{n}\right)$ be a commuting $n$-tuple of semi-normal operators. If $\operatorname{Sp}(T) \subseteq \mathbb{R}^{n}$ then $T_{j}$ is self-adjoint for each $j, j=1, \ldots, n$.

Proof. Suppose that $T_{j}=A_{j}+i B_{i}, B_{j} \neq 0$ for some $j$. Then there exists a real number $\mu_{j} \neq 0$ such that $\mu_{j} \in \sigma\left(B_{j}\right)$. Then by the above theorem, there exists $\lambda_{j} \in \mathbb{R}$ such that $\lambda_{j}+i \mu_{j} \in \sigma\left(T_{j}\right)$. See also [5]. But, by the projection property of Taylor's joint spectra, we have $P_{j}(\operatorname{Sp}(T))=\sigma\left(T_{j}\right)$, where $P_{j}$ is the projection onto the $j$ th co-ordinate. Thus we have $\lambda_{j}+i \mu_{j} \in \sigma\left(T_{j}\right)=P_{j}(\operatorname{Sp}(T))$. This contradicts the fact that $\mathrm{Sp}(T) \subseteq \mathbb{R}^{n}$. Thus $T_{j}$ is self-adjoint for each $j, j=1, \ldots, n$. 
In the passing, we remark that our proof of the theorem also gives a simpler proof of Putman's theorem [Lemma 3].

ACKNOWLEDGEMENTS. This research was partially supported by NSERC Grant No. A07545 and was done while the first author was visiting the Department of Mathematics and Statistics, University of Guelph, Canada, Guelph during the period September 20 to November 1, 1982.

Furthermore, the first author would like to take this opportunity to thank the Department of Mathematics and Statistics, University of Guelph for the hospitality he received during his stay.

\section{REFERENCES}

1. S. K. Berberian, Approximate proper vectors, Proc. Amer. Math. Soc. 13 (1962, 111-114).

2. M. D. Choi and C. Davis, The spectral mapping theorem for joint approximate point spectrum, Bull. Amer. Math. Soc. 80 (1974), 317-321.

3. R. E. Curto, Fredholm and invertible $n$-tuples of operators. The deformation problem, Trans. Amer. Math. Soc. 266 (1981), 129-159.

4. A. T. Dash, Joint spectra, Studia Math. 45 (1973), 225-237.

5. A. T. Dash, A note on joint approximate point spectrum, Mimeographed lecture notes, University of Guelph and Indiana University, 1972.

6. C. R. Putnam, On the spectra of semi-normal operators, Trans. Amer. Math. Soc. 119 (1965), 509-523.

7. C. R. Putnam, Commutation properties of Hilbert space operators and related topics (Springer, 1967).

8. J. L. Taylor, A joint spectrum for several commuting operators, J. Func. Anal. 6 (1970), 172-191.

JOETSU UNIVERSTTY OF EDUCATION

Department of MATHEMATICS

JOETSU 943

JAPAN

Department of Mathematics and Statistics

UNIVERSITY OF GUELPH

Guelph, Ontario, Canada 